\title{
Intracranial Dural Based Malign Mesenchymal Neoplasm: Case Report
}

Burcu Yapar Taşkoylu*, Mevci Ozdemir, Nagihan Yalcın, Murat Gokden, Yılmaz Kıroglu, Mustafa Korucu, Feridun Acar, Gokcen Demiray, Serkan Degirmencioglu, Gamze Gokoz Dogu and Arzu Yaren

Department of Internal Medicine, Faculty of Medicine, Division of Medical Oncology, Pamukkale University, Denizli, Turkey

"Corresponding author: Burcu Yapar Taşkoylu, Department of Internal Medicine, Faculty of Medicine, Division of Medical Oncology, Pamukkale University, Denizli, Turkey, Tel: + 05055807559; E-mail: drburcuyapar@gmail.com

Received date: May 10, 2014, Accepted date: Jun 10, 2014, Publication date: June 30, 2014

Copyright: (c) 2014 Taşköylü BY, et al. This is an open-access article distributed under the terms of the Creative Commons Attribution License, which permits unrestricted use, distribution, and reproduction in any medium, provided the original author and source are credited.

\begin{abstract}
Dural based sarcoma is a rare aggressive neoplasm arising from the multipotent primitive mesenchymal stem cells of the dura. A 61-year-old male patient presented with complaints of loss of visual acuity in his right eye for 1 week. Neuroimaging revealed a tumour located at the right temporal lobe. The mass was dural-based. Three years ago dural based lesion was seen. In that period radiological diagnosis was meningioma. Within three years, the lesion has grown and radiologically presumed meningioma view has been disappeared. After surgical resection of the mass pathological diagnosis of primary dural based sarcoma was made. As in soft tissue sarcomas, diagnosis is mainly based on light microscopy, while immunohistochemistry can improve accuracy of diagnosis. In this article we would like to highlight two things: one is dural sarcoma and meningioma cannot be distinguished radiologically, and the other is the patient's three-year history.
\end{abstract}

Keywords: Dural neoplasm; Differential diagnosis; Nonmeningotelial tumours

\section{Introduction}

Sarcomas involving the meninges may arise directly from mesenchymal cells or may develop within meningiomas. Sarcomas may also present by direct extension from the skull base, cranial vault or sinuses. Leptomeningeal metastases of extracranial soft tissue sarcomas are also possible. Symptoms depend upon the location and size of the tumour and can include headache, seizures, vomiting, somnolence or spinal cord compression. They usually represent hipointensity on T1 weighted and hyperintensity on T2 weighted MR images [1]. The incidence of meningeal sarcoma is difficult to assess. They are certainly rare tumors, probably constituting about 1 percent or less of intracranial tumours [2]. WHO classification of mesenchymal tumours are: tumours of the adipose tissue; including lipoma, liposarcoma, angiolipoma, fibrous and fibrohistiocytic tumours; solitary fibrous tumour, fibrosarcoma, and malignant fibrous histiocytoma, myogenic tumors; leiomyoma, leiomyosarcoma, rhabdomyoma, and rhabdomyosarcoma, osteocartilaginous tumors; chondroma, osteoma, osteochondroma, and chondro sarcoma and vascular tumors; for example hemangioma, epithelioid hemangioblastoma, and angiosarcoma [3].

\section{Clinical Presentation}

A 61-year-old male patient presented with complaint of loss of visual acuity in his right eye for a week. Physical and neurological examinations showed no abnormality in cranial nerves. The laboratory results, including blood count, liver and renal function, were within the normal range. MRI revealed tumour located at right temporal lobe which has been caused the shift. Three years ago dural based lesion was seen. In that period radiological diagnosis was meningioma (Figures 1 and 2). Within three years, the lesion grow and radiologically presumed meningioma view has been disappeared
(Figures 3 and 4). A craniotomy was performed and the mass was resected. Postoperative recovery was uneventful, there were no surgical complications, CT scan detected a small residual tumour (Figure 5). Pathological diagnosis was primary dural based sarcoma. Histologically, areas of necrosis around the pseudopapillary structures and increased cellularity showing the development of malignant tumor proliferation (Figure 6). By immunohistochemistry; vimentin, MBP (myelin basic protein), EMA (epithelial membrane antigen) (Figure 7), CD10 were positive in most cells. However, the tumor cells were negative for GFAP (glial fibrillary acidic protein), PANCK, Progesteron, RCC, Sinaptofizin, Norofilament, CK7, CK20, CEA, S-100 ve HMB-45, PAS, Aktin, Desmin, CD34, CD57. Ki-67 proliferation index $\% 40, \mathrm{p}^{53}(+)$. High power fields 2 mitosis was observed so there is minimal mitotic activity. Reticulin histochemical stainning reticular fibers individually wrap tumour cells (Figure 8). Leptomeningeal metastases of extracranial soft tissue sarcomas are also possible but there was no primary lesion was determined in PET CT and MRI of the abdomen. Gamma Knife was applied to the residual lesion. After gamma-knife, anthracycline and ifosfamid based chemotherapy was administrated. The patient is still on chemotherapy protocol.

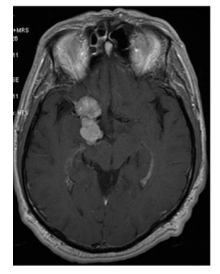

Figure 1: Contrast enhanced T1-weighted axial MR images shows initially homogenous and strong contrast enhanced extra-axial solid menengioma appearance with dural-tail. 
Citation: Taskoylu BY, Ozdemir M, Yalcin N, Gokden M, Kiroglu Y, et al. (2014) Intracranial Dural Based Malign Mesenchymal Neoplasm: Case Report. J Nucl Med Radiat Ther 5: 175. doi:10.4172/2155-9619.1000175

Page 2 of 4

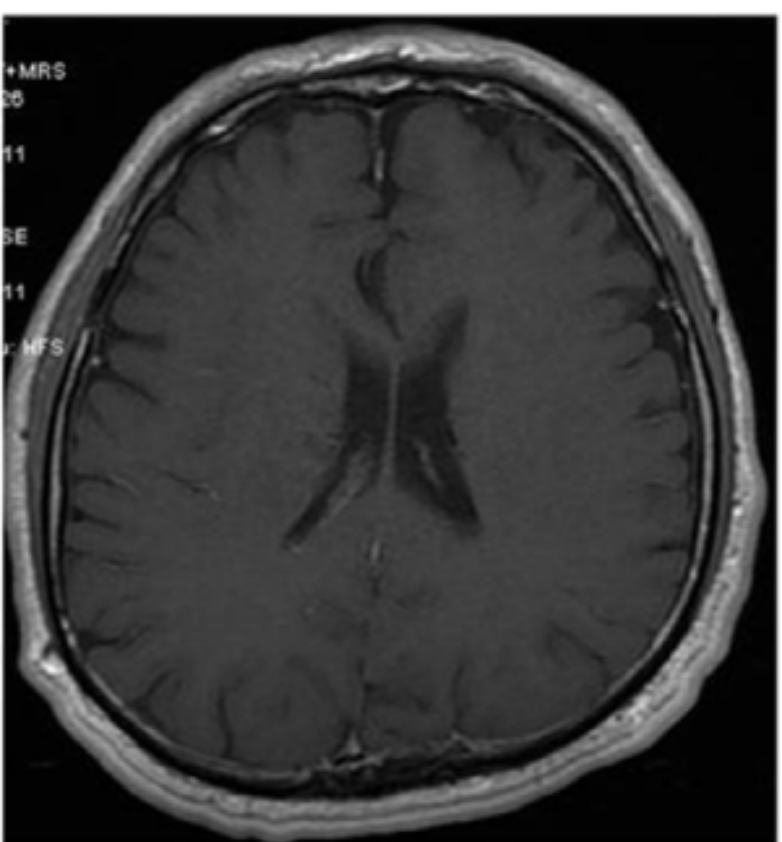

Figure 2: Contrast enhanced T1-weighted axial MR images shows no apparent mass appearance on upper areas.

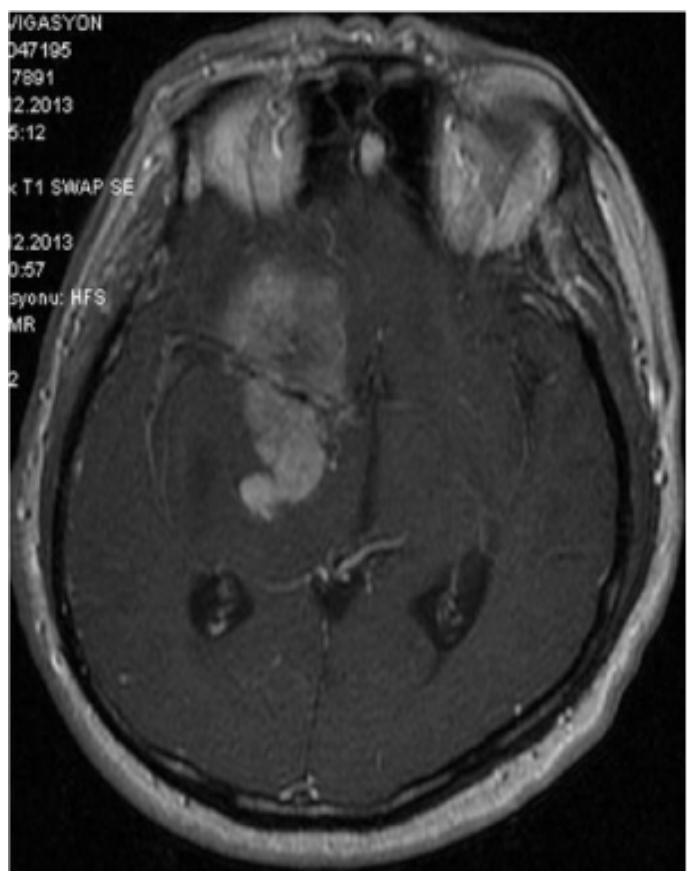

Figure 3: Contrast enhanced T1-weighted axial MR images 2 years later, pre-op images reveal the grown up mass with cystic-necrotic central.

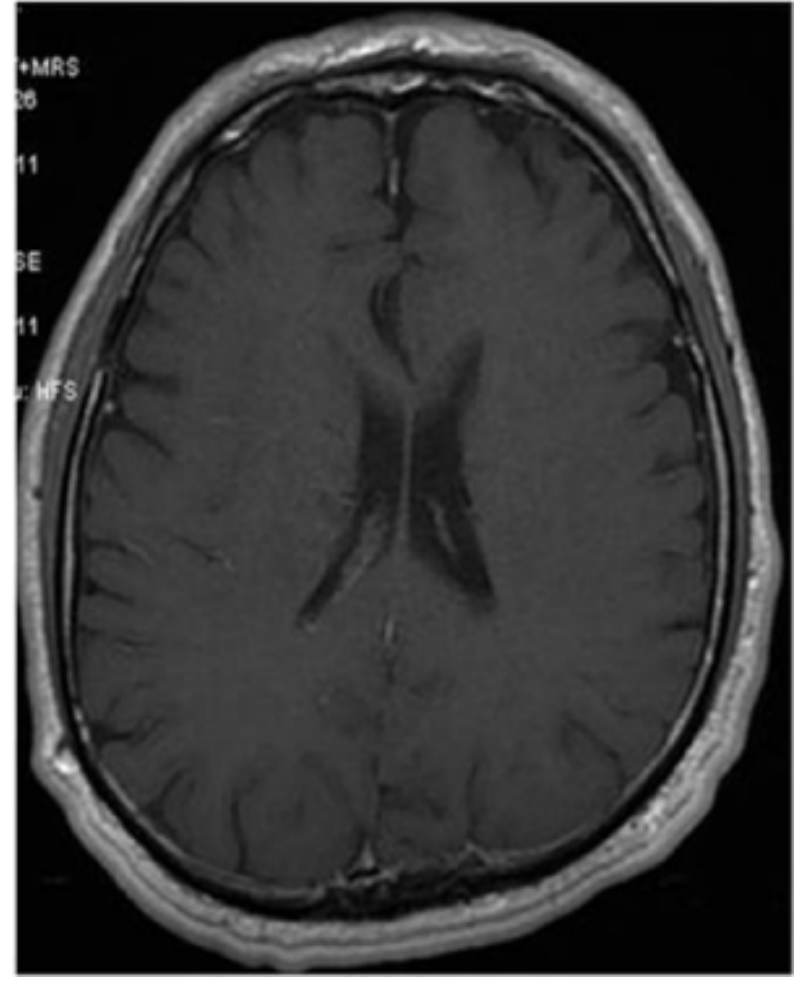

Figure 4: Contrast enhanced T1-weighted axial MR images 2 years later, pre-op images reveal the grown up mass with cystic-necrotic central.

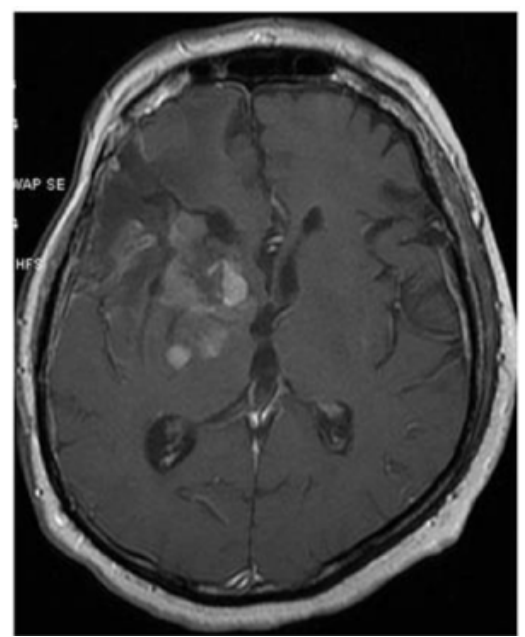

Figure 5: Contrast enhanced T1-weighted axial MR images post-op image reveals some residual areas. 
Citation: Taskoylu BY, Ozdemir M, Yalcin N, Gokden M, Kiroglu Y, et al. (2014) Intracranial Dural Based Malign Mesenchymal Neoplasm: Case Report. J Nucl Med Radiat Ther 5: 175. doi:10.4172/2155-9619.1000175

Page 3 of 4

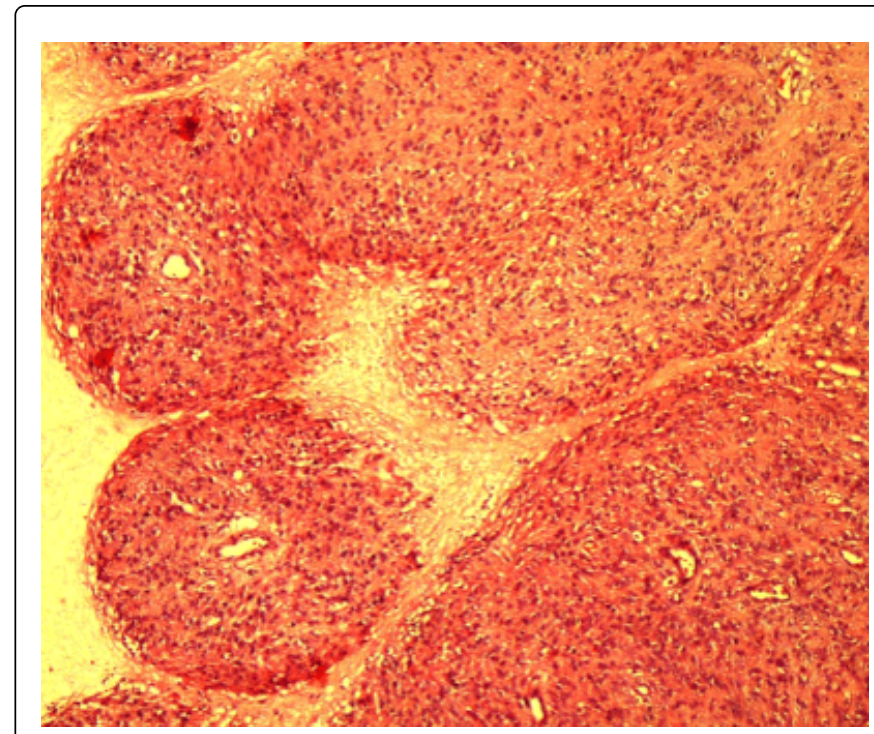

Figure 6: Areas of necrosis around the pseudopapillary structures (H\&E; 100X).

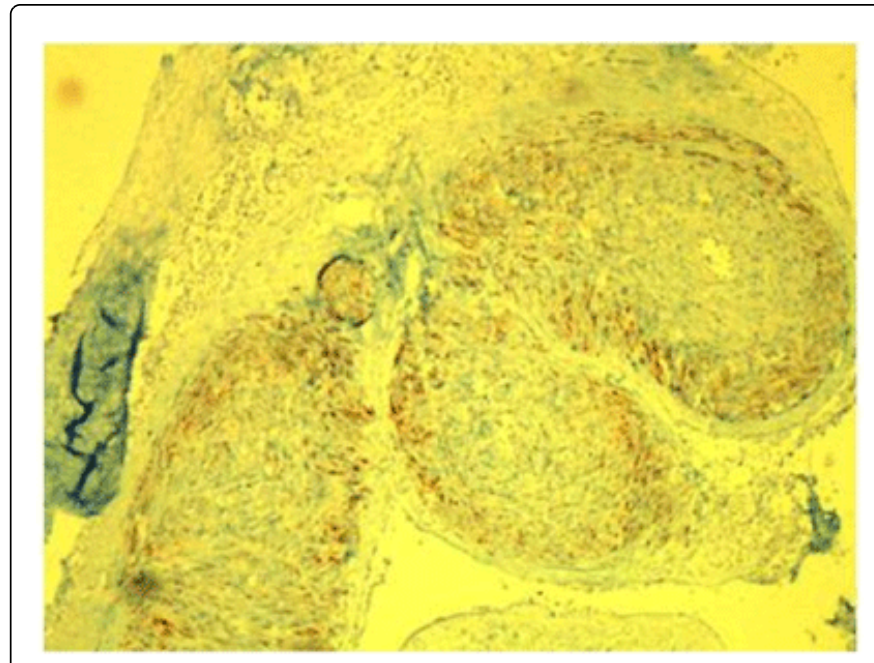

Figure 7: Immunohistochemical staining: positivity for $\operatorname{EMA}(200 \mathrm{X})$.

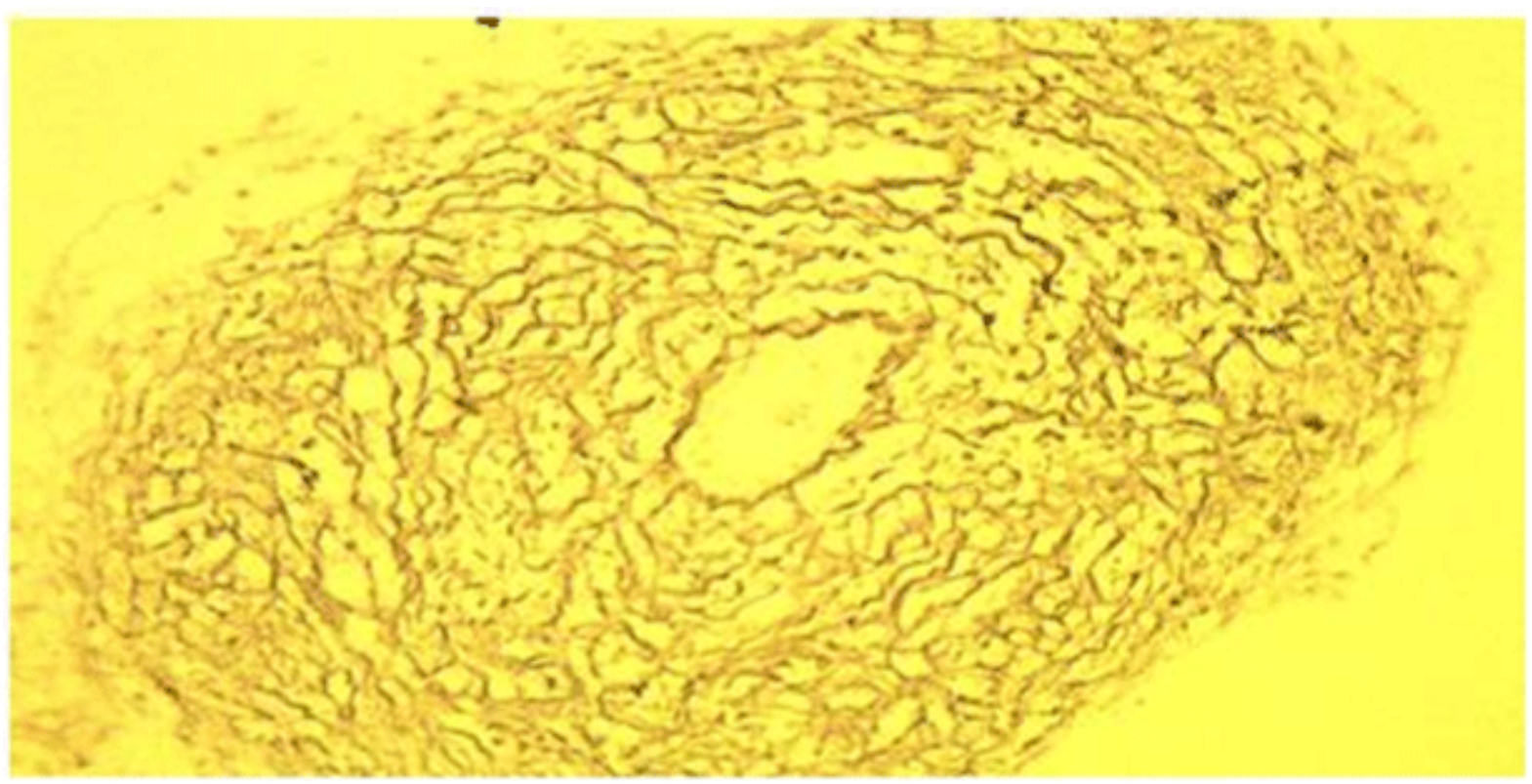

Figure 8: Immunohistochemical staining: positivity for Reticulin network (Reticulin; 400X).

\section{Discussion}

Meningeal sarcomas cause symptoms through a slow increase in size, either by compressing adjacent structures or by increasing intracranial pressure. Cranial mass had been discovered three years ago. At that time, the patient complained of headache. On MRI, tumor was well-circumscribed, lobular, homogeneously enhancing masses with broad dural attachments that were indistinguishable from meningioma [1]. Tumor have grown in three years and patient presented with complaints of loss of visual acuity in his right eye.
They may be differentiated along several lines, including solitary fibrous tumor, fibrosarcoma, malign fibrous histiocytomas, leiomyosarcomas, rhabdomyosarcoma. Solitary fibrous tumours have a characteristic immunohistochemical profile of CD34, vimentin, and Bcl-2 positivity, which is used in distinguishing these tumors from fibrous meningioma and other tumours that contain fibrous elements [4]. Malignant fibrous histiocytoma are usually of high histologic grade and have a high rate of local recurrence [5].

Treatment includes surgery and RT, with or without adjuvant chemotherapy. Treatment of meningeal sarcomas has been 
Citation: Taskoylu BY, Ozdemir M, Yalcin N, Gokden M, Kiroglu Y, et al. (2014) Intracranial Dural Based Malign Mesenchymal Neoplasm: Case Report. J Nucl Med Radiat Ther 5: 175. doi:10.4172/2155-9619.1000175

Page 4 of 4

unsatisfactory, despite the addition of RT and chemotherapy to surgery [6,7].

\section{Conclusion}

Nonmeningothelial mesenchymal tumors were rare seen. In our patient dural based lesion was seen three years ago. At that time, tumour indistinguishable from meningioma on MRI. İn a three years period radiologically presumed meningioma view has been disappeared. Sarcoma diagnosis has been made after the mass was resected. Gamma Knife was applied to the residual lesion. After gamma-knife anthracycline and ifosfamid based chemotherapy was given. An aggressive surgical resection is the best treatment of dural based sarcoma, because of the scarce response to radiotherapy and chemotherapy.

\section{References}

1. Ghosal N, Dadlani R, Gupta K, Furtado SV, Hegde AS (2012) A clinicopathological study of diagnostically challenging meningioma mimics. J Neurooncol 106: 339-352.
2. Ostrom QT, Gittleman H, Farah P, Ondracek A, Chen Y, et al. (2013) CBTRUS statistical report: Primary brain and central nervous system tumors diagnosed in the United States in 2006-2010. Neuro Oncol 15: 2.

3. Louis DN, Ohgaki H, Wiestler OD, Cavenee WK, Burger PC, et al. (2007) The 2007 WHO classification of tumours of the central nervous system. Acta Neuropathol 114: 97-109.

4. Ironside JW (2002) Diagnostic Pathology of Nervous System Tumors. Churchill Livingstone, New York.

5. Mevci Özdemir, Onur Ozgural, Melih Bozkurt, Fuat Mehmet Torun, Aylin Okcu Heper, et al. (2012) Primary Intracerebral Malignant Fibrous Histiocytoma Mimicking a Meningioma. Turkish Neurosurgery 22: 475-477.

6. Berger MS (2005) Textbook of neuro-oncology.Elsevier Saunders, Philadelphia.

7. Uluc K, Arsava EM, Ozkan B, Cila A, Zorlu F, et al. (2004) Primary leptomeningeal sarcomatosis; a pathology proven case with challenging MRI and clinical findings. J Neurooncol 66: 307. 